\title{
Universality of the lateral and angular distributions of electrons in large extensive air showers
}

\author{
Andrzej Śmiałkowski* \\ E-mail: asmial@kfd2.phys.uni.lodz.pl \\ Maria Giller \\ Department of Physics and Applied Informatics, University of Lodz, Poland
}

Based on shower simulations we show that the electron distribution $f(\theta, \varphi, r, E ; s)$, describing fully the fraction of electrons with energy $E$, at shower age $s$, at the distance from the axis $r$ and having angles $\theta, \varphi$, is the same for any shower, independently of the primary energy or mass and shower fluctuations. We find an analytic description of this function fitting it best in most populated regions of the variable space.

The 34th International Cosmic Ray Conference,

30 July- 6 August, 2015

The Hague, The Netherlands

\footnotetext{
* Speaker.
} 


\section{Introduction}
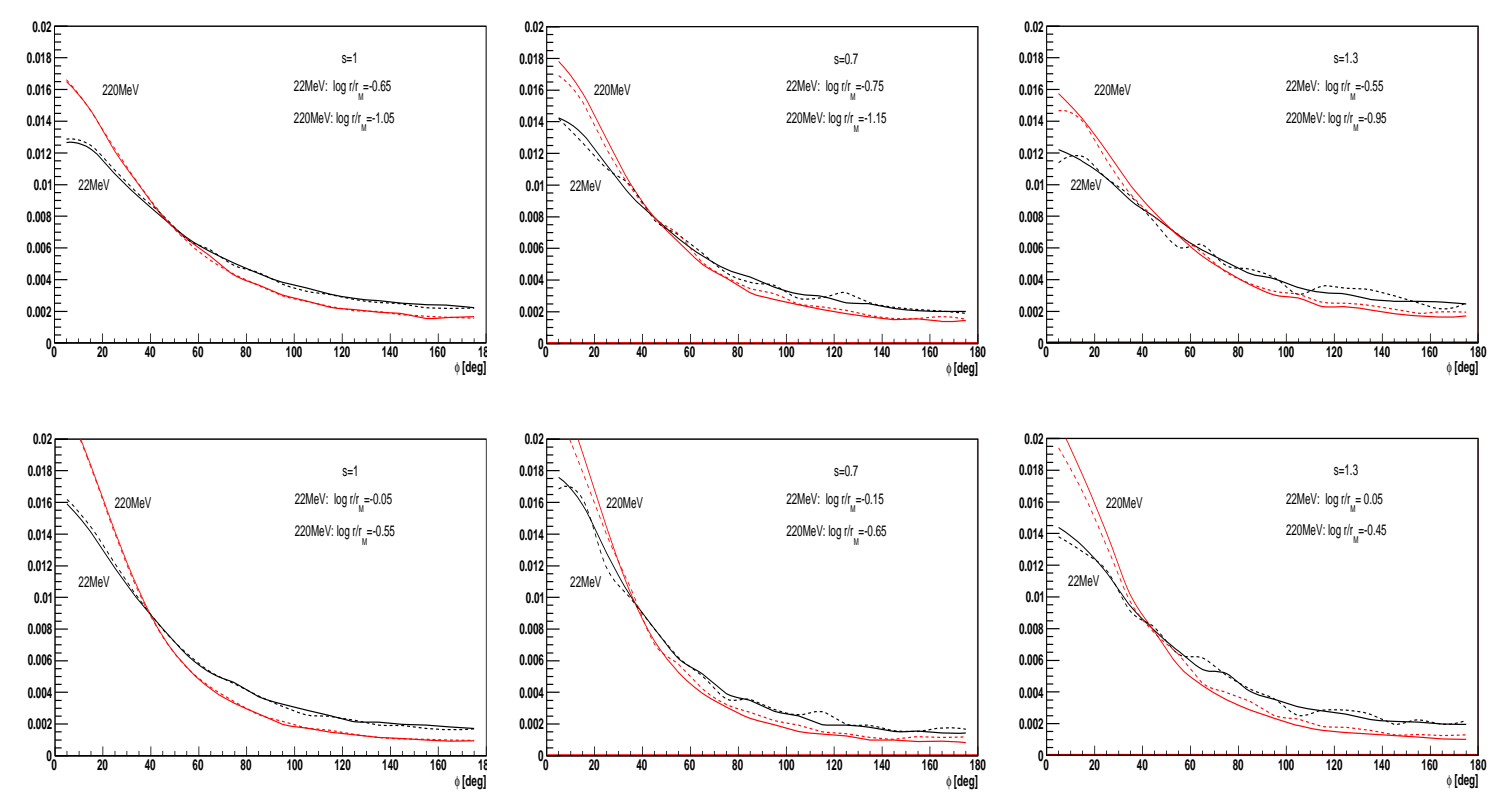

Figure 1: Distributions $f_{\varphi}\left(\varphi ; r / r_{M}, E, s\right)$ of electron azimuth angle $\varphi$ with respect to the shower axis for two electron energies $E$ within $\Delta E / E=0.15$, at two lateral distances $r / r_{M}$ within $\Delta \log \left(r / r_{M}\right)=0.1$. Continuous curve - one primary proton with $E_{0}=10^{19} \mathrm{eV}$, dashed - average of 10 iron showers with $E_{0}=10^{17} \mathrm{eV}$. Left column: $s=1$; middle: $s=0.7$; right: $s=1.3$. Independence of the primary particle's energy or mass is seen. $\varphi=0$ means directions away from shower axis.

In this paper we study various distributions of electrons (both signs) in large $\left(E_{0}>10^{16} \mathrm{eV}\right)$ extensive air showers (EAS), at different levels of shower development. These are the angular distributions, at different lateral distances $r$ from the shower core, for electrons of a given energy $E$. It was already shown $[1,2]$ that the electron energy distributions depend on the shower age $s$ only; they do not depend on the energy or mass of the primary particle. In [3] it was also shown that the lateral distributions of electrons with different energies depend only on the shower age, being independent of the primary particle. Moreover, they can be described by a single, universal function for any shower age [5]. We studied there [5] the distributions of electron's radial angles (in the shower axis - lateral vector plane), but integrated over the tangential angles, i.e. those in the plane perpendicular to the lateral vector, (and the other way round) and gave an analytical description of them for any electron energy $E$ and lateral distance $r$ (in $\mathrm{g} \mathrm{cm}^{-2}$ or in Molière radius $\left.r_{M}\right)$.

The aim of this paper is twofold: Firstly, to show a universality, in the sense of the independence of the primary particle and of the shower to shower fluctuations, of any electron distribution in a large shower. Secondly, to give a full description, in a possibly compact form, of the state of electrons in a shower.

\section{Universality of electron distributions}

The state of electrons in a shower is uniquely determined by the numbers $\Delta N_{e}(\theta, \varphi, r, E ; s)$ 

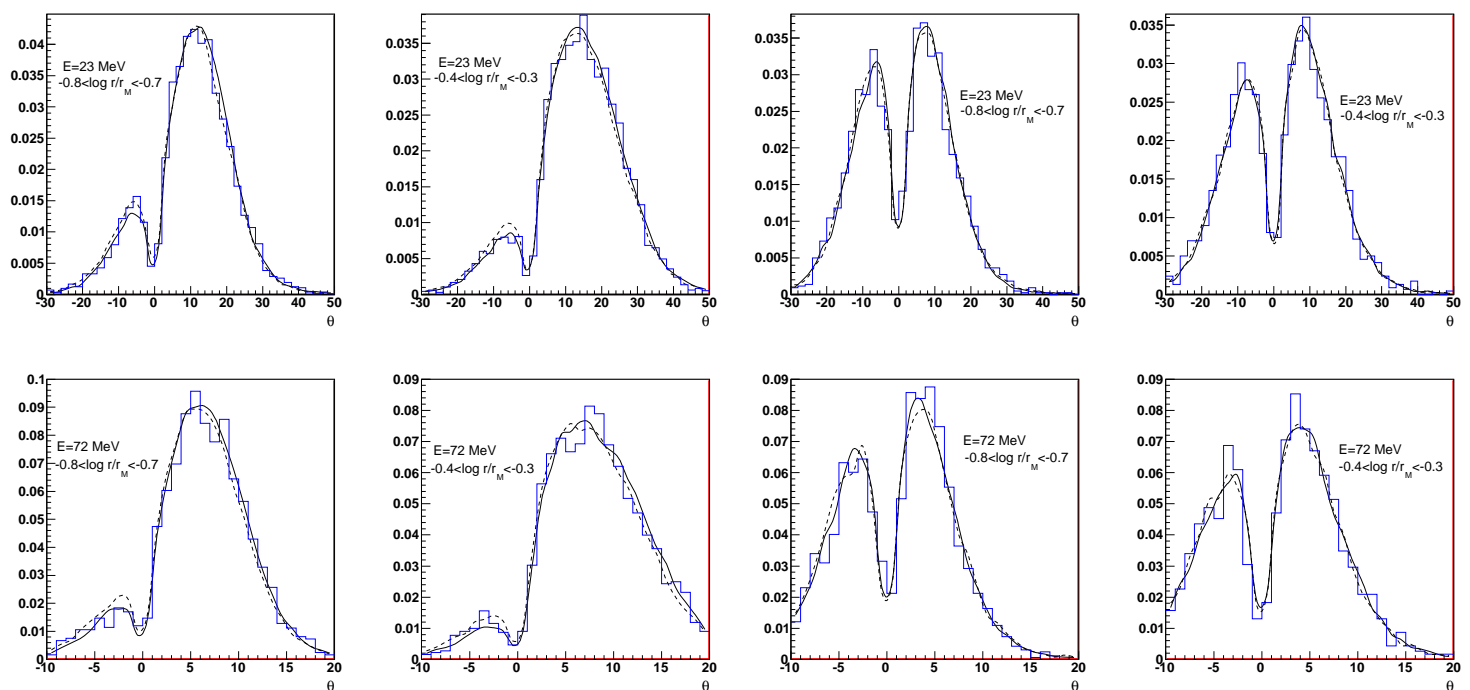

Figure 2: Distributions $f_{\theta}\left(\theta ; \varphi, r / r_{M}, E, s\right)$ of electron angle $\theta$ (in deg) for $s=1$ for two electron energies $E$ and two distances $r / r_{M}$. Continous curve - p $10^{19} \mathrm{eV}$, dashed - averaged of $10 \mathrm{x} \mathrm{Fe} 10^{16} \mathrm{eV}$, histogram - one Fe $10^{16} \mathrm{eV}$. Left half: $0<\varphi<20^{\circ}$ - bigger peaks, $160^{\circ}<\varphi<180^{\circ}$ - smaller peaks. Right half: $70^{\circ}<\varphi<90^{\circ}$ - bigger peaks, $90^{\circ}<\varphi<110^{\circ}$ - smaller peaks.
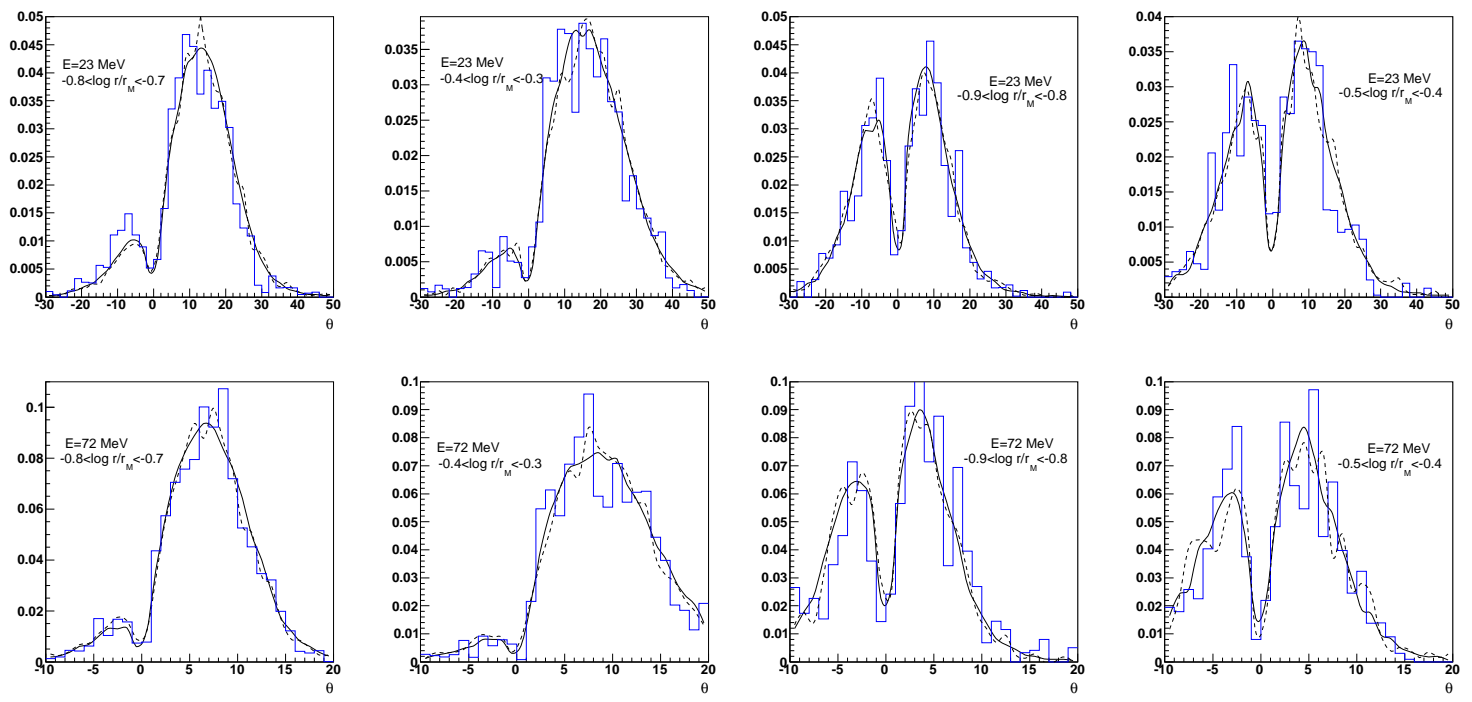

Figure 3: The same as in Fig.2 but $s=0.7$ and Fe showers are at $10^{17} \mathrm{eV}$.

given for all possibly occupied regions of the five variables. Each $\Delta N_{e}$ is the number of electrons with angles to the shower axis $(\theta, \theta+\Delta \theta)$, with azimuthal angle with respect to the axis $(\varphi, \varphi+$ $\Delta \varphi)$, at a lateral distance to the axis $(r, r+\Delta r)\left(\right.$ in $\left.g_{c m}^{-2}\right)$, with energy $(E, E+\Delta E)$, and at some shower age $s$. We define a function $f(\theta, \varphi, r, E ; s)$ as follows:

$$
\Delta N_{e}(\theta, \varphi, r, E ; s)=N_{e}(s) f(\theta, \varphi, r, E ; s) \Delta \theta \Delta \varphi \Delta r \Delta E
$$

where $N_{e}(s)$ is the total number of electrons at level $s$. Note that the function $f(\theta, \varphi, r, E ; s)$ has 

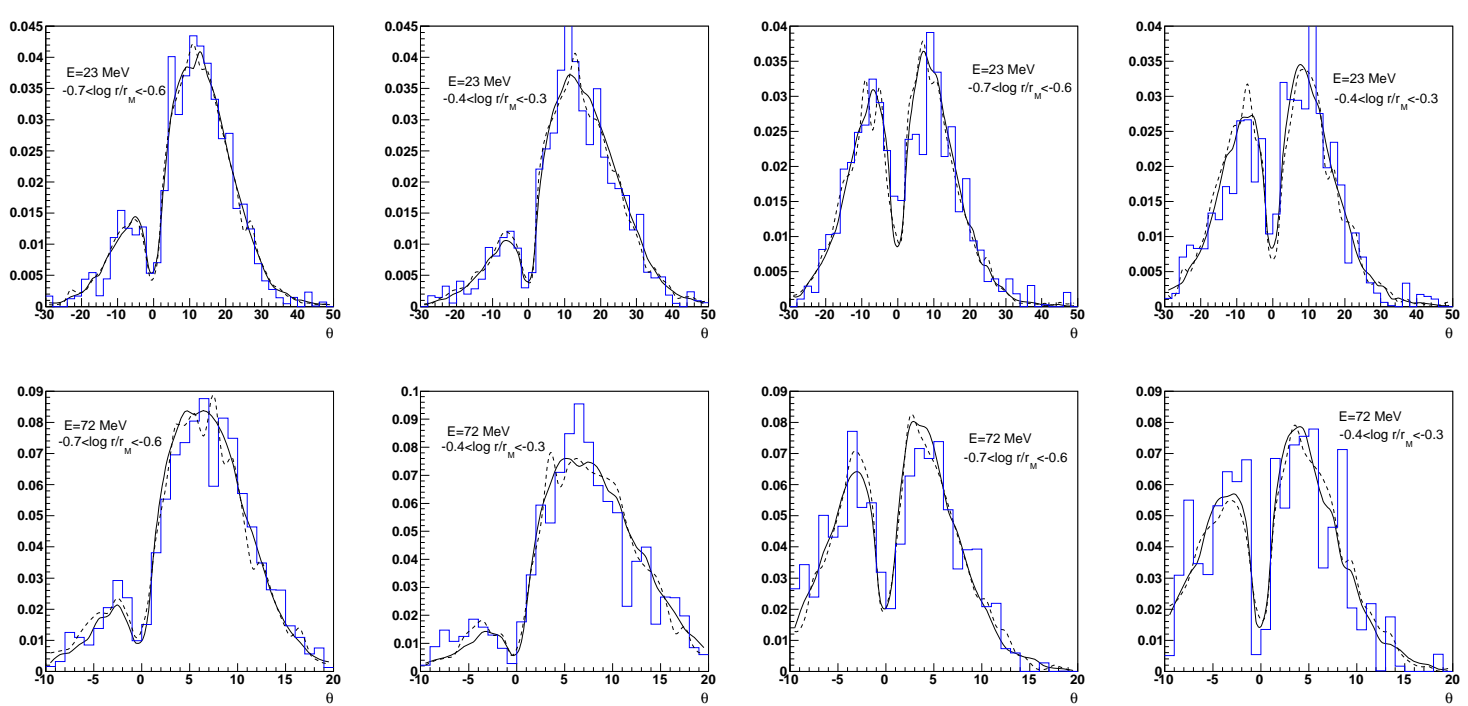

Figure 4: The same as in Fig.3 but $s=1.3$.

been defined for a single shower. However, we will show that for large showers, i.e. such that the numbers $\Delta N_{e}$ are large (>100), this function is universal. This would mean that it is independent of the primary particle energy or its mass, or even of the fluctuations in the shower development.

It is not difficult to show that $f$ can be represented as a product of functions, each depending on one variable and some number of parameters, as follows:

$$
f(\theta, \varphi, r, E ; s)=f_{E}(E ; s) f_{r}(r ; E, s) f_{\varphi}(\varphi ; r, E, s) f_{\theta}(\theta ; \varphi, r, E, s) .
$$

The variables on the r.h.s. of the semicolons are the parameters of the functional dependence on the variables before the semicolons. The functions $f_{E}, f_{r}, f_{\varphi}$ and $f_{\theta}$ are the normalised to unity. They are distributions : of electron energy $E$ at a given $s$, of distance $r$ at given $E$ and $s$, of the angle $\varphi$ at given $r, E$ and $s$, and of $\theta$ at given $\varphi, r, E$, and $s$ - respectively.

The energy distributions at various ages $f_{E}(E ; s)$ have been found already [1] and parametrised in an elegant way by Nerling et al [2]. The lateral distribution $f_{r}\left(r / r_{M} ; E, s\right)$ has also been shown to be universal [3, 4]. Moreover, in [5] it was shown that $f_{r}\left(r / r_{M} ; E, s\right)$, a function of three variables, can be represented as a function of only one variable.

It is seen from Eq.2.2 that to prove the universality of any electron distribution, represented by $f(\theta, \varphi, r, E ; s)$, it is left to demonstrate that each of the functions $f_{\varphi}(\varphi ; r, E, s)$ and $f_{\theta}(\theta ; \varphi, r, E, s)$ is universal.

To obtain the electron distributions we simulate showers with CORSIKA [6], find the number of electrons $\Delta N_{e}\left(\theta, \varphi, r / r_{M}, E, s\right)$ in variable bins $\Delta \theta \Delta \varphi \Delta\left(r / r_{M}\right) \Delta E$ at various ages $s$. (Note that $r / r_{M}$ is proportional to $r$ in $\mathrm{g} \mathrm{cm}^{-2}$ ). We restrict ourselves to variable regions where there are most electrons in the shower, i.e. to $0.7<s<1.3$ and $20 \mathrm{MeV}<E<200 \mathrm{MeV}$. Showers with $E_{0}=10^{17} \mathrm{eV}$ and $10^{16} \mathrm{eV}$ were fully simulated i.e. without the thinning procedure, whereas at $10^{19} \mathrm{eV}$ the thinning was, of course, used.

Fig.1 demonstrates the universality of the distributions $f_{\varphi}\left(\varphi ; r / r_{M}, E, s\right)$ of the azimuth angle $\varphi$ of electrons with two fixed energies $E=22 \mathrm{MeV}$ and $220 \mathrm{MeV}$, at two distances $r / r_{M}$ for each $E$, at 
three shower development levels $s=1,0.7$ and 1.3.

The distributions are shown for one proton shower with $E_{0}=10^{19} \mathrm{eV}$ and for an average of 10 iron showers with $E_{0}=10^{17} \mathrm{eV}$ (actually the fluctuations from shower to shower for $E_{0}=10^{17} \mathrm{eV}$ were not large, but for comparison with the proton shower it is better to flatten them out by averaging over some number of them). We see that essentially there is no difference between the two curves in any of the graphs of Fig.1. The particular values of $E$ and $\log \left(r / r_{M}\right)$ have been chosen in such a way as to correspond to two values on both sides of the maximum of the $\log E$, and correspondingly $\log \left(r / r_{M}\right)$, distributions (the latter describes fraction of electrons in rings with a constant thicknes $\left.\Delta \log \left(r / r_{M}\right)=0.1\right)$. At maxima the independence of the primary particle characteristics is even better.

Next, we go to the last function, $f_{\theta}\left(\theta ; \varphi, r / r_{M}, E, s\right)$, to be checked with respect to the universality - the distribution of angles $\theta$ for fixed values of the parameters $\varphi, r / r_{M}, E, s$. Figs 2,3 and 4 illustrate the independence of the angular distributions of electrons $f_{\theta}\left(\theta ; \varphi, r / r_{M}, E, s\right)$ of the primary particle energy and mass. The chosen regions of the azimuth angle correspond to the electrons deflected mostly away from the shower axis: $0<\varphi<20^{\circ}$, toward it: $160^{\circ}<\varphi<180^{\circ}$, and to those deflected perpendicularly to $\vec{r}$ : $70^{\circ}<\varphi<90^{\circ}$ and $90^{\circ}<\varphi<110^{\circ}$.

The average curve from 10 iron showers with the primary energy $E_{0}=10^{17} \mathrm{eV}\left(10^{16} \mathrm{eV}\right.$ for $s=1$ ) agrees very well with that referring to a single proton shower with $10^{19} \mathrm{eV}$. Comparing the average distribution with that for a single shower, both for $E_{0}=10^{17} \mathrm{eV}\left(10^{16} \mathrm{eV}\right.$ for $\left.s=1\right)$, one can also see fluctuations in individual bins which are due to small electron numbers in the bins. We have checked that the function stays practically the same down to $E_{0}=10^{16} \mathrm{eV}$, although the relative fluctuations are about 3 times larger than those in $10^{17} \mathrm{eV}$ showers.
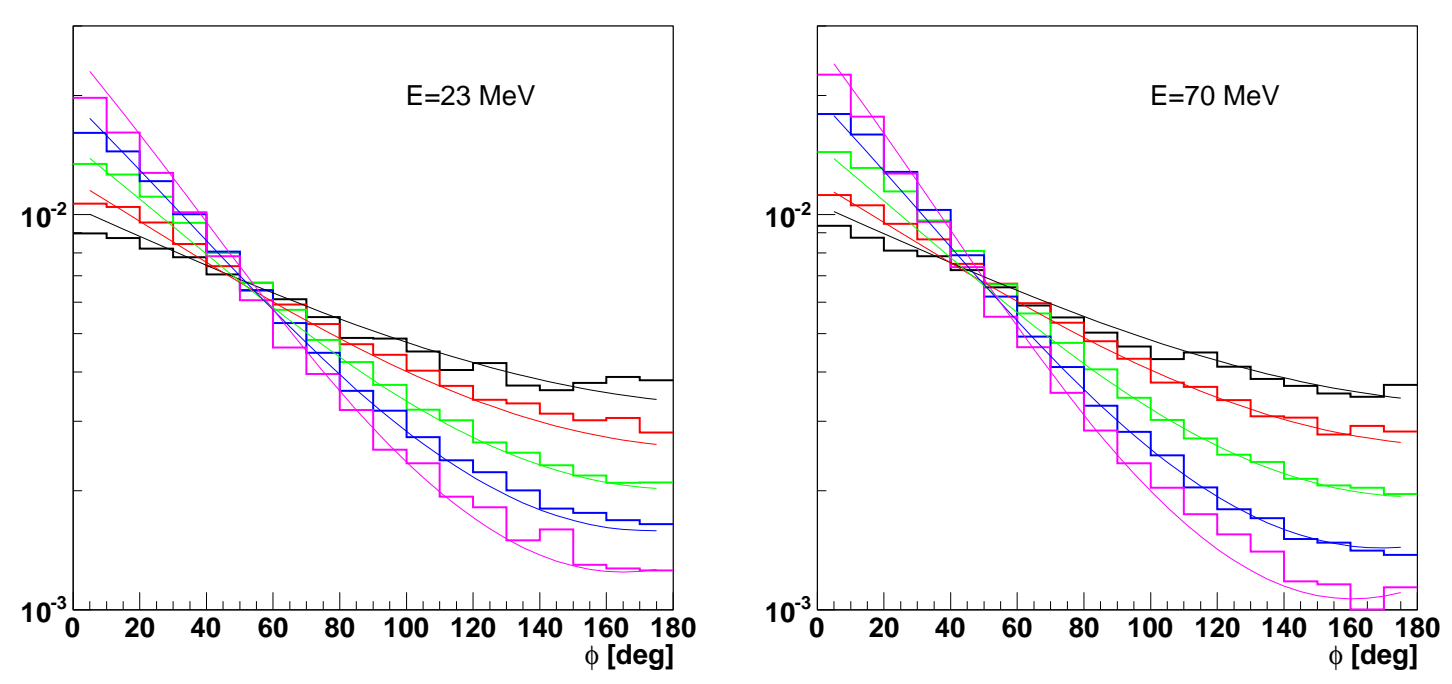

Figure 5: Comparison of actual (histograms) and parametrised (lines) distributions $f_{\varphi}\left(\varphi ; r / r_{M}, E, s=1\right)$ of azimuth angles $\varphi$ for two electron energies $E=23 \mathrm{MeV}$ and $E=70 \mathrm{MeV}$, each for five values of $r / r_{M}$; left graph (starting from the flattest): $\log r / r_{M}=-1.6,-1.1,-0.6,-0.1,0.4$; right graph: $\log r / r_{M}=$ $-1.8,-1.3,-0.8,-0.3,0.2$. One iron shower with $E_{0}=10^{17} \mathrm{eV}$ (without thinning). 


\section{Describing the universal distributions}

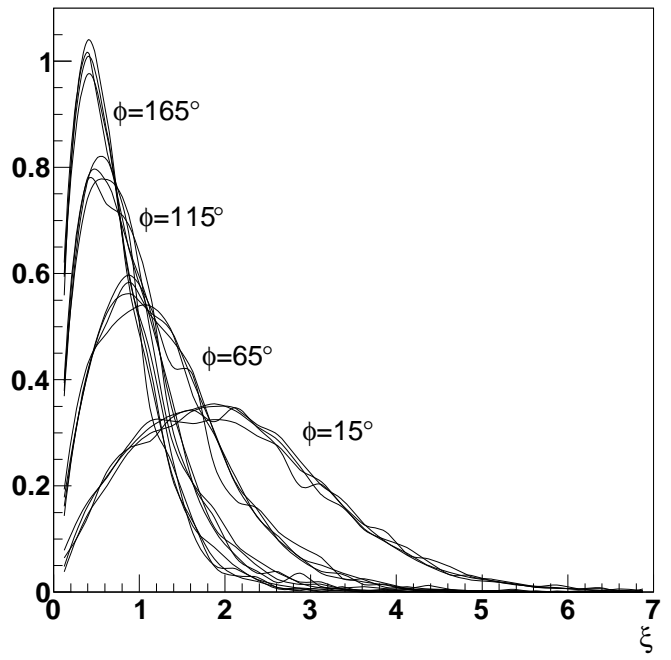

Figure 6: Independence of $F_{\xi}(\xi ; \varphi, s=1)$ of electron energy $E$ or distance $r / r_{M}$ for fixed $\xi$ (\$3.2). One iron shower with $E_{0}=10^{17} \mathrm{eV}$ (without thinning).

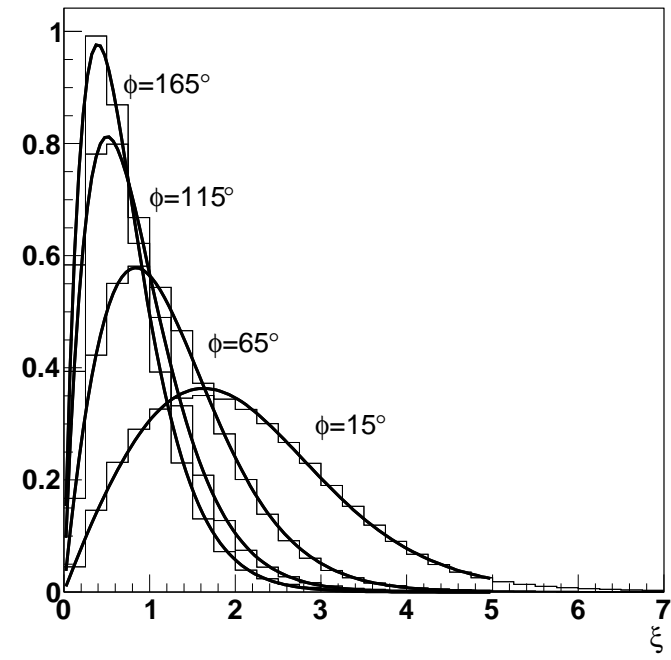

Figure 7: Comparison of the actual (histograms) and parametrised (lines) distributions $F_{\xi}(\xi ; \varphi, s=$ 1) referring to those of axial angle $\theta$. The same shower as in Fig.6.

The universality of the electron distributions allows one to undertake the task of finding their analytical description. We have tried to find a smaller number of variables on which the functions $f_{\varphi}(\varphi ; r, E, s)$ and $f_{\theta}\left(\theta ; \varphi, r / r_{M}, E, s\right)$ depend. For example, we succeded in doing so by representing $f_{r}\left(r / r_{M} ; E, s\right)$ as a function of just one variable $u=r / r_{E, s} \sim r / r_{M} E^{0.53} \exp ^{-0.89(s-1)}$ [5]. The results presented below concern shower maximum levels $s=1$, although some information concerning $s \neq 1$ will be given.

\subsection{Description of the distribution $f_{\varphi}\left(\varphi ; r / r_{M}, E, s=1\right)$ of azimuth angles $\varphi$.}

The histograms in Fig.5 present examples of the distributions $f_{\varphi}\left(\varphi ; r / r_{M}, E, s=1\right)$ for $E=23$ and $70 \mathrm{MeV}$ respectively, for several lateral distances $r / r_{M}$ (different in each case) in a logarithmic step (see figure captions). In the log-lin scale the distributions look more or less like a fraction of the cosine graph. Thus, we describe them in the form

$$
\log f_{\varphi}=A+B \cos (a \varphi+b)
$$

where the parameters $A, B, a$ and $b$ have been fitted for four values of electron energy: $E(M e V)=$ 23,39,70 and 124 ; and for each energy $E$ - for five values of $\log \left(r / r_{M}\right)$ in the most populated regions. The best fitting linear description is the following ( $\varphi$ in degrees):

$$
\begin{aligned}
& A=-2, \\
& B=(0.76+2.6 \cdot E)+(0.19+E) \cdot \log \left(r / r_{M}\right), \\
& a=(0.63+1.18 \cdot E)+(0.13+0.32 \cdot E) \cdot \log \left(r / r_{M}\right), \\
& b=68.8(1-E)+12.6 \cdot \log \left(r / r_{M}\right) \quad \text { where } E \text { is in } G e V .
\end{aligned}
$$


In Fig. 5 the lines represent function (3.1) with the parameters described by (3.2). We can see that the description does not deviate from histograms by more than $10 \%$. For $E>150 \mathrm{MeV}$ it does so, so a more complicated description of the parameters $A, B, a$ and $b$ would be needed. Unfortunately, in this case we have not succeeded in finding less than three variables to describe $f_{\varphi}$.

3.2 Description of the distribution $f_{\theta}\left(\theta ; \varphi, r / r_{M}, E, s=1\right)$ of axial angles $\theta$.

We have found a new variable $\xi$, defined as

$$
\xi=\frac{E^{\alpha} \theta}{\left(r / r_{M}\right)^{\beta}}, \text { where } \alpha>0 \text { and } \beta>0,
$$

such that $f_{\theta}\left(\theta ; \varphi, r / r_{M}, E, s\right)$ reduces to a function $F_{\xi}(\xi ; \varphi, s)$ of three, instead five, variables: $\xi, \varphi$ and $s$. We choose $E$ in $G e V$ and $\theta$ in degrees. Fig. 6 illustrates that it is actually the case. It shows $F_{\xi}(\xi ; \varphi, s)$ for four values of $\varphi$, at $s=1$. For each $\varphi$ there are four curves, referring to four combinations of two electron energies: $E=23$ and $70 \mathrm{MeV}$ and two lateral distances: $\log \left(r / r_{M}\right)=-1.4$ and -0.7 . It is seen that the distributions are essentially independent of energy or distance once the value of $\xi$ is fixed. However, the parameters $\alpha$ and $\beta$ depend on $\varphi$ as follows:

$$
\begin{aligned}
& \alpha=0.615+1.12 \cdot 10^{-3} \cdot \varphi-2.4 \cdot 10^{-6} \cdot \varphi^{2} \text { and } \\
& \beta=0.29-1.8 \cdot 10^{-3} \cdot \varphi+4.2 \cdot 10^{-6} \cdot \varphi^{2} \quad \text { where } \varphi \text { is in degrees. }
\end{aligned}
$$

We fit the distributions $F_{\xi}(\xi ; \varphi, s=1)$ for various $\varphi$ in bins $\Delta \varphi=10^{\circ}$, with a function

$$
F_{\xi}(\xi ; \varphi, s=1)=\frac{C \xi}{\left(d+e^{\xi}\right)^{\gamma}}
$$

The parameters $d$ and $\gamma$ depend on $\varphi$, as it is obvious from Fig.6, and so does $C$ that should normalise the integral $\int F_{\xi}(\xi ; \varphi, s=1) d \xi$ to unity. However, in Fig.7 it is the histograms which are normalised to unity and the three parameters $C, d$ and $\gamma$ have been fitted independently. We have found that

$$
\begin{aligned}
\log d & =1.06-0.011 \cdot \varphi \text { for } 20^{\circ}<\varphi<150^{\circ}, \\
& =0.84 \text { for } \varphi<20^{\circ} \\
& =-0.59 \text { for } \varphi>150^{\circ} \\
\gamma & =1.32-0.45 \cdot \log \varphi+0.54 \cdot \log ^{2} \varphi
\end{aligned}
$$

We have checked that for the best fitted parameters the integrals deviate from unity by less than $2 \%$ The best fitted curves are shown, together with actual distributions, in Fig. 7. It can be seen that the description by formula (3.5) is quite satisfactory.

\section{Summary}

In this work we have demonstrated the universality of both angular distributions: $f_{\varphi}\left(\varphi ; r / r_{M}, E, s\right)$ and $f_{\theta}\left(\theta ; \varphi, r / r_{M}, E, s\right)$, of electrons with various energies $E$, at various lateral distances $r / r_{M}$ and at various shower ages $s$. 
Together with the universality of the electron energy distributions and of their lateral distributions this allows us to claim that any electron distribution in a large shower is universal. This claim concerns mainly the variable regions corresponding to the bulk of electrons in a shower. A quantification of some deviations of the distribution $f(\theta, \varphi, r, E ; s)$ for variable regions away from the most populated needs further work.

We have also parametrised the angular distributions for $s=1$. In the case of the distribution of the axial angle $\theta$ we have found a variable $\xi=E^{\alpha} \theta\left(r / r_{M}\right)^{-\beta}$ such that $f_{\theta}\left(\theta ; \varphi, r / r_{M}, E, s=1\right)$ depends actually on only three, instead of five, variables.

For $s \neq 1$ the distributions do not differ much from those at $s=1$. However, since the differences reach sometimes more than $10 \%$, an additional dependence of parametrisations on age $s$ needs to be done.

Acknowledgements This work has been supported by the grant no. DEC-2013/10/M/ST9/00062 of the Polish National Science Centre.

\section{References}

[1] M. Giller, G. Wieczorek, A. Kacperczyk, H. Stojek and W. Tkaczyk, J. Phys. G: Nucl. Part. Phys. 30 (2004) 97

[2] F. Nerling, J. Blümer, R. Engel and M. Risse, Astroparticle Phys, 24 (2006) 421

[3] M. Giller, H. Stojek, G. Wieczorek, Int. Journal of Modern Phys. A, Vol 20, (2005), No 29, p. 6821

[4] S. Lafebre, R. Engel et al, Astroparticle Phys. 31 (2009), 243

[5] M. Giller, A. Śmiałkowski and G. Wieczorek, Astroparticle Phys. Vol 60, (2015), p. 92-104

[6] D. Heck, J. Knapp et al., Technical Report 6019 (1998) Forchungszentrum, Karlsruhe 